Bulletin de la Société Royale des Sciences de Liège, Vol. 85, 2016, p. 181 - 186

\title{
An Adaptive Super-Resolution Algorithm Applied to Magnetic Resonance Imaging
}

\author{
Kian JAFARI ${ }^{1}$ \\ Electrical Engineering Department, Shahid Beheshti University,Teheran, Iran
}

\begin{abstract}
Each year, many people are diagnosed with cancers such as brain cancers, worlwide. It is essential to have a short delay and high accuracy in diagnostic of fast spreading cancerous cells. Magnetic Resonance Imaging (MRI) is one of the important means in cancer diagnosis. In this context, various Super-Resolution (SR) methods have been proposed for the use in MRI scans after acquisition time. In this paper, an adaptive super-resolution algorithm is presented which can detect MRI scans defects and try to reconstruct them. As a consequence, the proposed SR algorithm may increase sensitivity and specificity of the output results. One of the important advantage of the proposed algorithm is its ability to maintain the specifications without any changs in significant part of the data. Thus, the proposed method can be emplyed in MRI for better results in real-time in terms of sensitivity and accuracy.
\end{abstract}

Keywords: Super Resolution Algorithm, Magnetic Resonance Imaging, cancerous cells

\section{Introduction}

It is estimated that in the year 2016, 78,000 people will be diagnosed with primary tumors of the brain and central nervous system. This estimation includes 4,000 children and teens with a brain or central nervous system tumor[2]. MRI, which uses magnetic fields and radio waves to create an image of the brain, has a significant role in medical diagnosis [3, 4]. Although, no health hazards have been detected in lower versions of MRI scanners, upgrading the machine in order to get better results can be costly and hazardous. Furthermore, increasing the time spent inside the MRI tube could be risky for claustrophobic patients and using strong magnetic fields increases headaches and dizziness after the scan [15].

Medical societies issue guidelines for when MRI scans should be used. In most cases, overuse is not recommended. Medical societies also often recommend other types of methods to be used for diagnosis when possible. One of the most common cases in which MRI scans are used is in locating brain tumors. When compared to the deployment of conventional methods, employing an algorithm that can be used in low resolution (low chemical) scans such as superresolution methods is more advantageous from different points of view, specifically regarding the patients' health $[11,12]$.

In this paper, we have originated methods in order to utilize the usage of the super resolution algorithm on MRI scans. This is less costly and time consuming when compared to hardware related methods, and the results are more interpretable and precise comparing to the original images [6]. The algorithm consists of the following stages: 1.registration, 2.interpolation, 3.Deblurring and 4.intelligent enhancement. The rest of this paper is organized as follows. In section 2, we briefly review the previous contributions in this field. In section 3, we describe the proposed Enhanced Super-resolution algorithm in high-level language. In section 4, some simulation results for a real MRI scan is illustrated and a comparison is made between the MRI scans before and after processing by the proposed algorithm. A conclusion is finallty driven in section 5 .

\section{Recent Contributions}

Enhancing MRI scan resolution comes at the cost of lower signal to noise ratio or longer acquisition time [11]. In order to prevent unnecessary complications and expenses that follow the usage of high field MRI scanners and complex hardware modifications, post-processing methods and algorithms have been developed and suggested to increase MRI scan resolution, diffusion or details [1,12]. The super-resolution methods have been utilized in this effect, and have been known to be beneficial when compared with conventional methods [11]. The super-resolution TDI, which is mainly used for scans in need of higher contrast levels, has been mentioned and used for MRI scans in perticular. The super- resolution TDI utilizes methods to enhance diffusion in order to help with tumor localization and discrimination.

${ }^{1}$ Email: $\underline{\text { k_jafari@ @bu.ac.ir }}$ 
A major problem with deploying post-acqusition methods and algorithms is that the processed output should not contain false data resulted from the methods deployed. In super-resolution TDI, false data in the processed data has been found. Unfortunately, the noisy outcome may mislead the doctors and cause serious misdiagnosis problems [13].

The methods deployed in this paper have the advantage of reaching high resolution and less severely defected imaging, intelligently and with minimal data modification. The previous works include image reconstruction via sparse representation which is a commonly used in statistical image modeling $[9,10,14]$.

\section{The Proposed Super Resolution Algorithm}

The main goal of this algorithm is to increase the resolution of an MRI scan in order to increase the details shown in the MRI scan; This results in discriminating the tumor from the healthy tissue. In order to increase the discrimination and localization of the tumor, in this paper we are using the Multi Frame in opposed to the Single Frame method. The stages of the algorithm is shown in Figure 1.

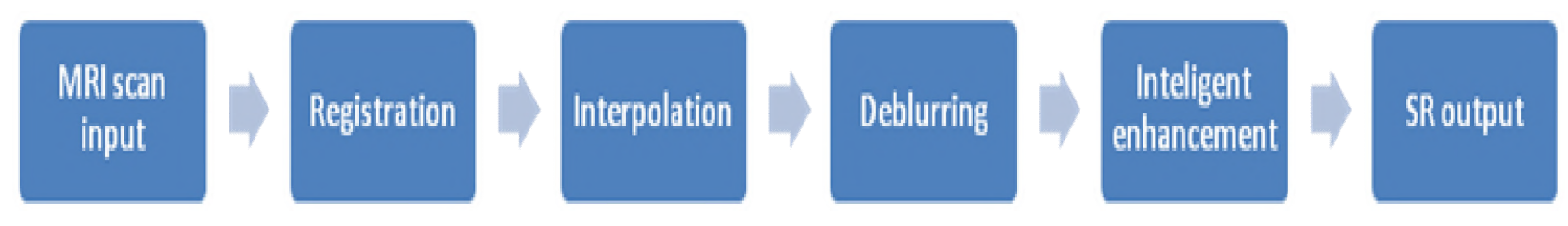

Figure 1. The proposed SR algorithm.

The first three stages are common in super resolution techniques, and therefore have been summarized. The fourth stage has been fully described as the proposed method to discriminate between colors and atomize image enhancement. Figure 3 shows and head MRI image, before and after of applying the algorithm.

\section{- Stage I and II. Saving the scan in order to align the frames with lower resolutions:}

In the simplest possible way, we choose the first frame as the reference frame. Then, we shift the remaining frames in the $\mathrm{X}, \mathrm{Y}$ and $\mathrm{XY}$ direction until we reach a point where the difference from the main frame reaches a minimum prespecified threshold. At the end of this stage, we have a shift with minimum difference with the reference for each frame. The result of this stage is an average of the shifts acquired from each frame and the reference frame.

\section{- Stage III. Interpolation in order to increase the resolution of the frame:}

In order to increase the speed of this algorithm, we can use basic interpolation algorithms, such as the nearest neighbor.

\section{- Stage IV. Smoothing to decrease noise:}

In case that the scan has too much noise, we have to use smoothing as well at this stage.

\section{- Stage V. Intelligent enhancement to discriminate the tumor from healthy tissue and simplify locolization:}

The intelligent enhancement has always been a challenge. The proposed algorithm, uses an efficient method to enhance the three defected frames, as shown in Figure 3, intelligently, and without any outer interference. The details are described in the following. 


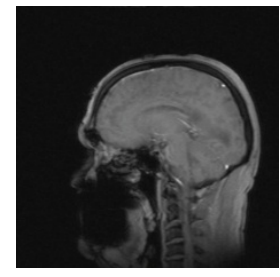

$\mathbf{a}$

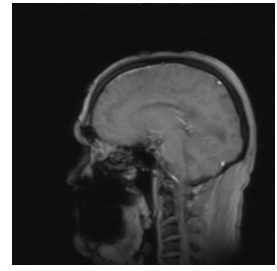

b

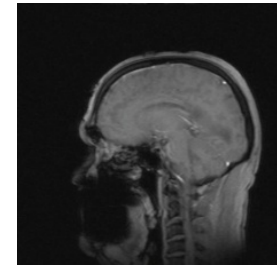

c

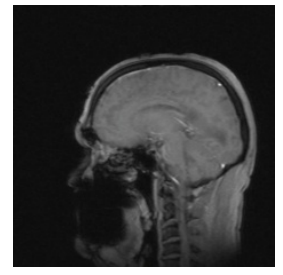

d

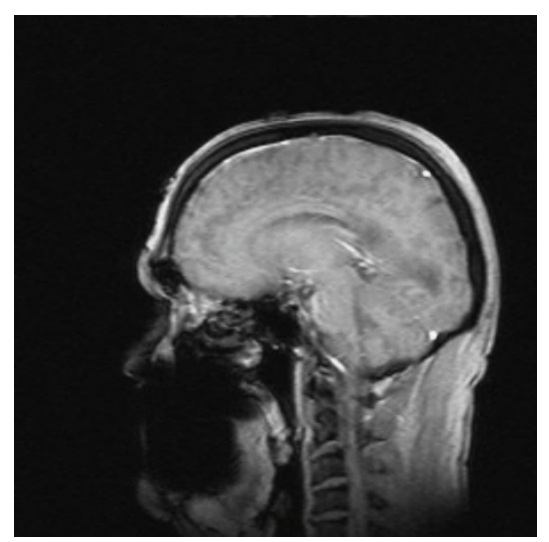

e

Figure 2. Frames (a) to (d) are taken from one scene and frame e is the final output of the algorithm.

First, we draw the histogram of the scans. Then, we divide it into three equal parts, horizontally. If the frequency of distribution is concentrated at the first part, it reveals that the pictures' lighting is too low. Otherwise, if the frequency of the distribution is concentrated in the middle as a mass, then the problem lies in the contrast of the scans and depicts that the contrast is too high. If the frequency of distribution is concentrated in the third part of the histogram, it shows that the lighting is too high. To correct the lightening defects, we firstly calculate the average light intensity of each component in RGB view. Each component for a pixel is calculated by (4):

$$
\begin{aligned}
& \text { new value }=\left(256 *\left(\frac{\text { old value }}{256}\right)^{\text {gamma1 }}+3 *\left(256 *\left(\frac{\text { old value }}{256}\right)^{\text {gamman }}\right) / 4\right. \\
& \text { if } \operatorname{menn} 3128: \text { gommo1 }=0.5+(\operatorname{mean} * 0.0039) \\
& \text { if mean } \leq 128: \text { gamma1 }=1.0+(\text { mean }-128) * 0.00156 \\
& g a m m a 2=\log \frac{125}{256} / \log \frac{\operatorname{masen}}{256}
\end{aligned}
$$

For frames with low contrast, the following method is developed using a large database of images. The components in RGB view must change from the first database: ' $x$ ' to ' $y$ '. The sample inputs and outputs for changing the curve (a diagram that maps inputs to outputs) is shown in (5):

$$
\begin{aligned}
& x=[0,14,15,28,41,53,65,80,99,117,137,152,167,181,193,205,217,223,240,255] \\
& y=[2,2,5,7,14,24,35,52,77,103,134,158,184,205,220,232,242,246,253,255]
\end{aligned}
$$

The relation between ' $x$ ' and ' $y$ ' is shown in Figure 3. By using (4) and values of ' $x$ ', ' $y$ ' in this figure, one can calculate each component in RGB mode:

$$
\text { new value }=\left(p_{1} * o v^{2}+p_{2} * o v^{2}+p_{2} * o v+p_{4}+\text { factor } *(o v-128)+128\right) / 2
$$

where,

ov is the old value,

$p_{1}=-3.693 \mathrm{e}-00$,

$p_{2}=0.01465$

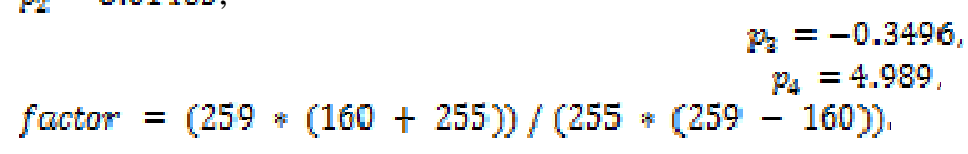


Bulletin de la Société Royale des Sciences de Liège, Vol. 85, 2016, p. 181 - 186
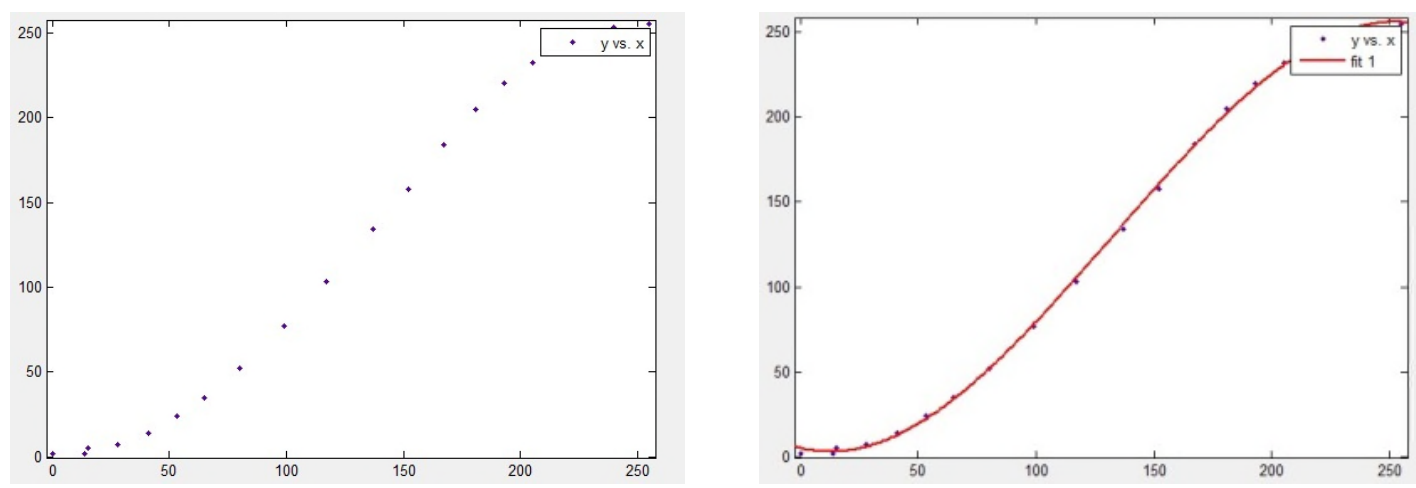

Figure 3. The relation between ' $x$ ' and ' $y$ '

\section{Simulation results}

By using the super resolution algorithm, we can use basic MRI imaging to diagnose a brain tumor at its early stages by highlighting the discrimination between the brains tissue and the tumor. This algorithm has the advantage of highlighting the details of defected or low resolution MRI scan frames. The results obtained from the proposed algorithm on the MRI scans are illustrated in Figure 4. The initial MRI scans are shown in figure 4-left and the achieved results can be seen in figure 4-right. As shown in this figure, the results show significant improvement in terms of light intensity and contrast from the initial scans, with minimal medical data modification. 

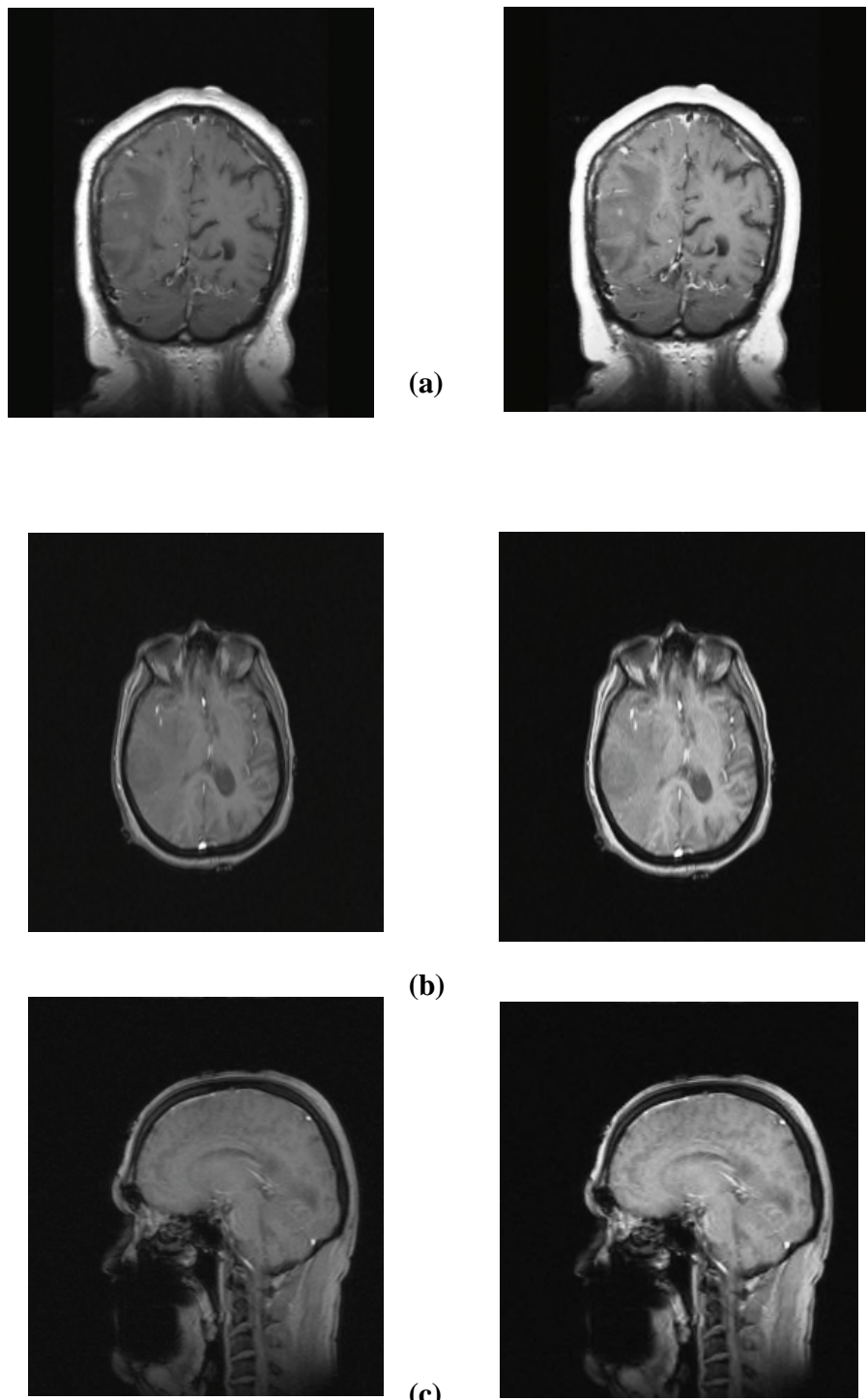

(b)

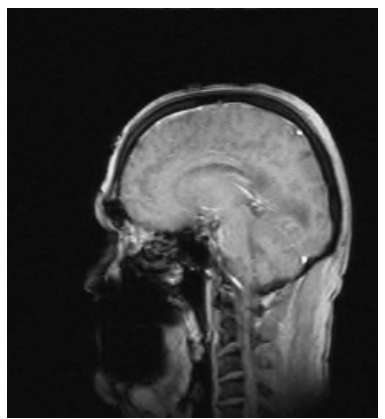

Fig 4. The MRI scans before (left) and after (right) applying the proposed algorithm

\section{Conclusions}

In this paper, an adaptive super-resolution algorithm is proposed for Magnetic Resonance Imaging (MRI) that is one of the key tools in cancer diagnosis. The proposed algorithm can detect MRI scans defects and try to reconstruct them. Thus, sensitivity and specificity of the output scan results may be increased by applying the proposed method. The ability to maintain the specifications of the images without any changs in significant part of the data is one of the advantages of the proposed technique. Simulation results showed that the use of the proposed method in MRI may increase the quality of MRI scans in terms of sensitivity and accuracy.

\section{REFERENCES}

[1] Y. Wang , J. Qiao , J. Li , P. Fu, S. Chu , J. F. Roddick, Sparse representation-based MRI super-resolution reconstruction, Journal of Measurement, Elsevier, 47, pp. 946-953, 2014.

[2]Statistics adapted from the American Brain Tumor Association, National Institute of Health and the American Cancer Society's publication, Cancer Facts \& Figures, 2016.

[3] B. A. Moffat, T. L. Chenevert, T. S. Lawrence, C. R. Meyer, T. D. Johnson, Q. Dong, C. Tsien, S. Mukherji, D. J. Quint, S. S. Gebarski, P. L. Robertson, L. R. Junck, A. Rehemtulla, B. D. Ross, Functional diffusion map: A 
noninvasive MRI biomarker for early stratification of clinical brain tumor response, PubMed, 102(15), pp. 5524-9, 2005 .

[4] W. Hollingworth, C.J. Todd, M.I. Bell, Q. Arafat, S. Girling, K.R. Karia, A.K. Dixon, The diagnostic and therapeutic impact of MRI: an observational multi-centre study, Clinical Radiology, Elsevier, 55 (11), pp. 825-31, 2000 .

[5] R.B. Schwartz, L. Hsu, D.F. Kacher, T.Z. Wong, E. Alexander, S. Okon, CR. Guttmann, P.M. Black, RA. Kelley, T. Moriarty, C. Martin, HG. Isbister, C.D. Cahill, S.A.Spaulding, F.A. Jolesz, Intraoperative dynamic MRI: localization of sites of brain tumor recurrence after high-dose radiotherapy, JMRI, Wiley, 8(5), pp. 1085-1098, 1998.

[6] R.C. Gonzalez, R.E. Woods, Digital Image Processing. Prentice Hall, Upper Saddle River, NJ 07458, 2002.

[7] T. Ogawa, M. Haseyama, Missing image data reconstruction based on adaptive inverse projection via sparse representation, IEEE Transactions on Multimedia, IEEE, 13(5), pp. 974-992, 2011.

[8] J. Yang, J. Wright, T. Huang, Image super-resolution via sparse representation, IEEE Transactions on Image Processing, IEEE, 19, pp. 2861-2873, 2010.

[9] E. Plenge D.H.J. Poot, M. Bernsen, G. Kotek, G. Houston, P. Wielopolski, L.Weerd, W.J. Niessen, E. Meijering, Super-Resolution Methods in MRI: Can They Improve the Trade-Off Between Resolution, Signal-to-Noise Ratio, and Acquisition Time? Magnetic Resonance in Medicine, Wiley, 68(6), pp. 1983-1993, 2012.

[10] H. Greenspan, G. Oz, N. Kiryati, S. Peled, MRI inter-slice reconstruction using super-resolution, Magn Reson Imaging, 20, pp. 437-446, 2002.

[11] F. Calamante, Robert E. Smith, J.D. Tournier, D. Raffelt, A. Connelly, Quantification of voxel-wise total fibre density: Investigating the problems associated with track-count mapping, NeuroImage, Elsvier, 17(15), PP. 284-293, 2015.

[12] A. Jegatheeswari. P MCET, T. Nadu, India, B. Amudha. J.C. Sudhakar, Sparse representation based image deblurring and super resolution, Advances in Engineering, Science and Management (ICAESM), 2012 International Conference on Date of Conference, pp. 30-31, March 2012

[13] M.J. Versluis, W.M. Teeuwisse, M. JP van Osch, A.G. Webb, Subject tolerance of 7 T MRI examinations, JMRI, Wily, 38(3), pp. 722-725, 2013. 